\title{
A hybrid systems framework for cellular processes
}

\author{
Kwang-Hyun Cho ${ }^{\mathrm{a}, *}$, Karl Henrik Johansson ${ }^{\mathrm{b}}$, Olaf Wolkenhauer ${ }^{\mathrm{c}}$ \\ ${ }^{a}$ College of Medicine, Seoul National University, Chongno-gu, Seoul 110-799, Korea \\ ${ }^{\mathrm{b}}$ Department of Signals, Sensors and Systems, Royal Institute of Technology, 10044 Stockholm, Sweden \\ c Department of Computer Science, University of Rostock, 18051 Rostock, Germany
}

Received 1 July 2004; accepted 5 December 2004

\begin{abstract}
With the availability of technologies that allow us to obtain stimulus-response time series data for modeling and system identification, there is going to be an increasing need for conceptual frameworks in which to formulate and test hypotheses about intra- and inter-cellular dynamics, in general and not just dependent on a particular cell line, cell type, organism, or technology. While the semantics can be quite different, biologists and systems scientists use in many cases a similar language (notion of feedback, regulation, etc.). A more abstract system-theoretic framework for signals, systems, and control could provide the biologist with an interface between the domains. Apart from recent examples to identify functional elements and describing them in engineering terms, there have been various more abstract developments to describe dynamics at the cell level in the past. This includes Rosen's (M,R)-systems. This paper presents an abstract and general compact mathematical framework of intracellular dynamics, regulation and regime switching inspired by $(\mathrm{M}, \mathrm{R})$-theory and based on hybrid automata.
\end{abstract}

(c) 2004 Elsevier Ireland Ltd. All rights reserved.

Keywords: Systems biology; Cellular processes; (M,R)-theory; Dynamical model; Hybrid automata

\section{Introduction}

The renewed interests in systems biology reflects the natural shift of focus occurring in modern life sciences: away from the discovery of new components and their molecular characterization, towards an understanding of functional activity, interactions and the organization

\footnotetext{
* Corresponding author. Present address: Korea Bio-MAX Institute, 3rd Floor, International Vaccine Institute (IVI), Seoul National University Research Park, San 4-8, Bongcheon 7-dong, Gwanak-gu, Seoul 151-818, Korea. Tel.: +82 2887 2650; fax: +82 28872692.

E-mail address: ckh-sb@snu.ac.kr (K.-H. Cho).
}

of components in higher structural levels (Wolkenhauer et al., 2003). As more stimulus-response, time series data become available, a need for a system-theoretic framework of cellular processes, emerges. There has been considerable progress in mathematical modeling of intracellular dynamics, employing either stochastic simulation (Paulsson, 2004; Rao et al., 2002) or nonlinear differential equations to model aspects of cell signaling (Kholodenko et al., 2002; Brightman and Fell, 2000; Schoeberl et al., 2002; Asthagiri and Lauffenburger, 2001; Cho and Wolkenhauer, 2003; Cho et al., 2003a,b,c; Tyson et al., 2003; Heinrich et al., 2002), gene expression or transcriptional control amongst oth- 
ers (see De Jong, 2002; Smolen et al., 2000; Rao et al., 2002 for survey articles). As alternatives to the dynamic systems approach there have been also various other techniques to model dynamics, rooted in formal languages, state machines, graph theory, artificial intelligence, etc. (Shmulevich et al., 2002; Friedman et al., 2000; Maki et al., 2001; Rzhetsky et al., 2004; Jordan, 1996). While in some cases parameters for models are directly identified from experimental data (Cho et al., 2003a; Swameye et al., 2003; Tegner et al., 2003) more often a model is tuned for its simulation to match the experience of the biologist. The validation with experimental data is then somewhat indirect. In any case, mathematical modeling and simulation is gaining acceptance as a complementary tool to test or generate hypotheses in molecular and cell biology. While the semantics can be quite different, biologists and control engineers use a similar language. A more abstract system-theoretic framework for signals, systems, and control could provide an interface between both conceptual domains. This would help translating common terminology, including "feedback", "control", "regulation", "amplification", "filtering", etc. Apart from recent examples to identify functional elements and describing them in engineering terms (Wolf and Arkin, 2003; Iglesias and Levchenko, 2002) there have been various more abstract formalisms to describe dynamics at the cell level in the past. These include Rosen's (M,R)-systems (Rosen, 1971; Casti, 1988; Wolkenhauer, 2001), which are revisited in the present paper.

While at the molecular level many processes appear to be of random nature, at higher levels of structural organization, i.e., cells, tissue, and organs, well-defined principles of functional relationships emerge. The basic physical building block is the cell, which through networks of biochemical reactions realizes many fundamental processes relevant to development and disease. Biochemical reaction networks are also referred to as 'pathways', which are the biologist's conceptual frameworks to organize system variables, i.e., to identify relevant genes, proteins, metabolites, and to characterize their interactions in relation to cellular functions, including cell division, cell death, cell cycle, differentiation, proliferation, etc. More specifically, these include from gene to cell level upwards: gene expression, transcription, translation, metabolism, physiological, and immunological response and control processes. Rosen recognized that many of these highly specific and complex functions can be characterized by two generic aspects: a transformation process, which he called 'metabolism' and a regulatory component, whose function he described as 'repair'. These two abstract components form the basis for the theory of $(\mathrm{M}, \mathrm{R})$ systems.

Inspired by $(\mathrm{M}, \mathrm{R})$-theory, this paper aims at developing an extended framework of intracellular dynamics, regulation and regime switching, based on hybrid automata (Lygeros et al., 2003; Alur and Dill, 1994; Alur et al., 2000). The theory of hybrid systems allows us to complement continuous dynamics, which form the basis for many pathway models, with other regulatory or response levels that include changes to the elementary structure of dynamics (regime change, switching, non-deterministic transitions, and so forth). The proposed framework also allows for sudden structural changes which, following Rosen's and Casti's terminology, we call 'mutation'. Mutation is a structural change within a gene, chromosome and protein resulting in changes to the dynamics at gene-, protein-, metabolite-, or the physiological level.

\section{2. (M,R)-theory for biological cellular processes}

Considering the functionality of a cell, Rosen and Casti identified a metabolic component, representing basic biochemical processes and a maintenance or $r e$ pair component, which ensures the cell's regular functioning in response to disturbances. Historically, the original formulation of $(\mathrm{M}, \mathrm{R})$-theory was first given by Rosen (1971) and it was further developed by Casti (1988). Following Casti's presentation (Casti, 1988), we consider first metabolic activity, represented by the mapping

$h: \Omega \rightarrow \Gamma$

where $\Omega$ is the set of environmental stimuli and $\Gamma$ is the set of cellular responses. We denote by $H(\Omega, \Gamma)$ the family of all these mappings, i.e., the set of all physically realizable metabolic processes. We consider then the following basal metabolism for normal operation:

$\gamma^{*}=h^{*}\left(\omega^{*}\right)$

where $\omega^{*}, h^{*}, \gamma^{*}$ denote the environmental input, the metabolic map, and the cellular output, respectively, 


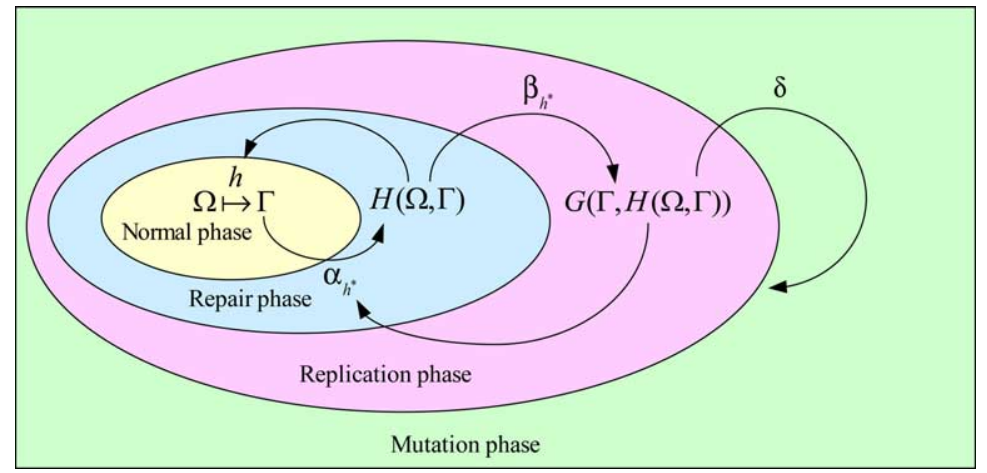

Fig. 1. Conceptual modeling of cellular processes. Each transition to a different phase or an alternative dynamic regime can be modeled as a discrete transition, which further invokes a hybrid system framework.

when everything is working according to plan under a normal condition. For deviations to the normal function, including external and internal disturbances to the cell's chemical activity, we introduce a repair map:

$\alpha_{h^{*}}: \Gamma \rightarrow H(\Omega, \Gamma)$

with the boundary condition $\alpha_{h^{*}}\left(\gamma^{*}\right)=h^{*}$. Since the repair component itself can be subject to disturbances, we require a further regulatory element, referred to as a replication map:

$$
\beta_{h^{*}}: H(\Omega, \Gamma) \rightarrow G(\Gamma, H(\Omega, \Gamma))
$$

with the boundary condition $\beta_{h^{*}}\left(h^{*}\right)=\alpha_{h^{*}}$, where $G(\Gamma, H(\Omega, \Gamma))$ denotes the family of all repair maps. We can summarize the abstract model structure for cellular processes in terms of the following morphisms:

$\Omega \stackrel{h^{*}}{\longrightarrow} \Gamma \stackrel{\alpha_{h^{*}}}{\longrightarrow} H(\Omega, \Gamma) \stackrel{\beta_{h^{*}}}{\longrightarrow} G(\Gamma, H(\Omega, \Gamma))$

with boundary conditions

$\alpha_{h^{*}}\left(\gamma^{*}\right)=h^{*} \quad$ and $\quad \beta_{h^{*}}\left(h^{*}\right)=\alpha_{h^{*}}$.

If the dynamics encoded by $h$, and its regulatory or supervisory components $\alpha_{h^{*}}(\cdot), \beta_{h^{*}}(\cdot)$, are not sufficient to cope with external disturbances or fluctuations, the next level of response is a transition to an alternative dynamic regime, for which we here introduce the discrete transition map $\delta$. The conceptual framework developed here is outlined in Fig. 1.

\section{Dynamical models and (M,R)-systems}

Before we proceed to develop a concrete structure of a cellular system, we first consider an extension of the (M,R)-description in terms of mappings, into a dynamical model familiar to control engineers. This can be done by shifting our focus from the relationship between conceptual mappings to the actual processes occurring in the cell. We now regard cellular processes as a dynamical system and focus on the inputs $\omega(t)$ : $\mathbb{R}_{+} \rightarrow \Omega \subset \mathbb{R}^{m}$ and $\lambda(t): \mathbb{R}_{+} \rightarrow \Lambda \subset \mathbb{R}^{r}$, the outputs $\gamma(t): \mathbb{R}_{+} \rightarrow \Gamma \subset \mathbb{R}^{p}$, and states $x(t): \mathbb{R}_{+} \rightarrow X \subset \mathbb{R}^{n}$. Note the abuse of notation in that $\Omega$ and $\Gamma$ are now subsets of Euclidean spaces, while in the (M,R)-model $\Omega$ and $\Gamma$ denote signal spaces. The dynamics of the cellular system is governed by:

$\dot{x}=f(x, \omega, \sigma)$

$\gamma=\zeta(x)$

where the environment variable $\sigma=\sigma(\lambda(t), e) \in \sum$ is a function of a time-varying external disturbance $\lambda(t) \in \Lambda$ and a constant internal control $e \in E$. Here, $\sum$ is the set of possible environment variables, which are depending on the cellular status and $E$ is the set of admissible internal controls. This dynamical model describes the cellular system in the normal operational phase. As indicated by the repair map of the (M,R)model, the cell may transit into other phases, which require repair and replication. This can be covered by a state-space model, introducing the partitioning of $X$. 


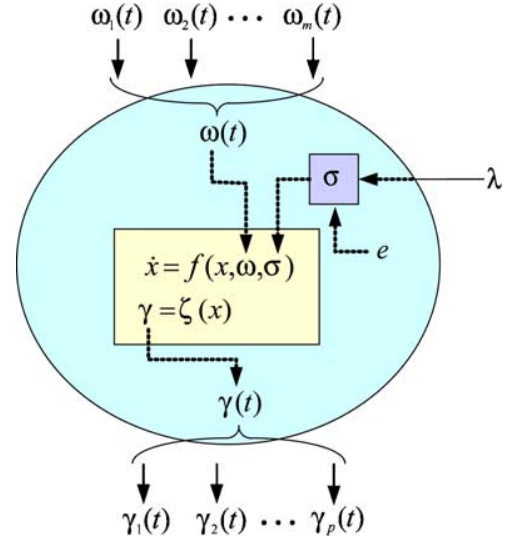

Fig. 2. A dynamical model of the cellular processes. The external disturbance $\lambda$ affects the environment variable $\sigma$ and it is further controlled by an internal control $e$. Transitions into different operating phases due to the external disturbances are accounted for partitioning the state-space into the corresponding disjoint subspaces.

into two disjoint subsets, $X_{\mathrm{d}}$ and $X_{\mathrm{ud}}$ which represent 'desirable' and 'undesirable' operating modes, respectively. Similarly, we define the corresponding output partitions: $\Gamma_{\mathrm{d}}=\zeta\left(X_{\mathrm{d}}\right)$ and $\Gamma_{\mathrm{ud}}=\zeta\left(X_{\mathrm{ud}}\right)$. The deviation from basal metabolism in normal operating phase is assumed to occur due to external disturbances and which affect the environment variable $\sigma$. The proposed dynamical model of the cellular processes is illustrated in Fig. 2.

\section{Hybrid automaton model of the cellular processes}

For the previous dynamical model to describe the possible state transitions taking place, we employ the semantics of hybrid automata (Lygeros et al., 2003; Alur and Dill, 1994; Alur et al., 2000). A hybrid automaton can capture the non-determinism of a state transition, which is convenient in modeling and analysis of highly uncertain cellular processes. In cellular processes, those state transitions corresponding respectively to repair, replication, and mutation are of course all triggered by the failure of a proper metabolism, but it is also non-deterministic whether a specific transition can occur for a given failure situation.

A hybrid automaton $Z$ is a collection $Z \triangleq(Q, X, f$, Init, $D, A, C, R)$, where $Q$ is the discrete state space, $X$ the continuous state space, $f: Q \times X \rightarrow X$ the vector field, Init $\subset Q \times X$ the set of initial states, $D$ : $Q \rightarrow P(X)$ the domain, $A \subset Q \times Q$ the set of arcs, $C$ : $A \rightarrow P(X)$ the guard condition, and $R: A \times X \rightarrow P(X)$ is the reset map. We refer to $(q, x) \in Q \times X$ as the state of $Z$. Roughly speaking, hybrid automata define possible evolutions for the state. Starting from an initial value $\left(q_{0}, x_{0}\right) \in$ Init, the continuous state $x$ flows as described by the vector field $f\left(q_{0}, \cdot\right)$, while the discrete state $q$ remains constant. Continuous evolution can go on as long as $x$ remains in $D\left(q_{0}\right)$. If at some point $x$ reaches a guard $C\left(q_{0}, q_{1}\right)$, for some $\left(q_{0}, q_{1}\right) \in A$, the discrete state may change to $q_{1}$. At the same time the continuous state is reset to some value in $R\left(q_{0}, q_{1}, x\right)$. After this discrete transition, continuous evolution resumes and the whole process is repeated. It is convenient to visualize hybrid automata as directed graphs $(Q, A)$ with vertices $Q$ and $\operatorname{arc} A$. With each vertex $q \in Q$, we associate a set of initial states Init $_{q}=\{x \in X \mid(q$, $x) \in$ Init $\}$, a vector field $f(q, \cdot)$ and domain $D(q)$. With each arc $a \in A$, we associate a guard $C(a)$ and a reset map $R(a, \cdot)$ For a non-autonomous system, we need to further include an external input $u \in U$ in $Z$, denoted by $Z_{u}$, and extend the above synopsis accordingly. A trajectory or solution of a hybrid automaton is called an execution or run. The definition of an execution involves conditions on the initial state, the continuous and discrete evolution. We say that a hybrid automaton accepts an execution or not (see Lygeros et al., 2003, for more formal definitions). It is important to note that a hybrid automaton may accept many executions or none from a single initial state, i.e., a hybrid automaton can be non-deterministic or blocking. Conditions for existence and uniqueness of executions are given in Lygeros et al. (2003).

There are numerous biological processes in which a multitude of dynamic regimes are employed to ensure the functioning of the cell. These are not necessarily 'regulatory' elements but also decision processes with permanent consequences. For example, during the development of an organism, organs, or tissue, cells differentiate, i.e., they adopt a specialized biochemical and/or physiological role. There are therefore two kinds of change in genome activity (Alberts et al., 2002; Brown, 1999): transient or switch-like, reversible responses to external stimuli of the cell via signaling compounds that either enter the cell or act through binding to surface receptors; secondly irreversible changes of genome activity underlying 
differentiation, and which can be brought about by DNA rearrangements, changes in chromatin structure, and positive feedback loops. Immunoglobulins are proteins that help protect an organism against invasion by bacteria, viruses, and other unwanted substances by binding to these antigens. This binding is very specific so that every antigen is recognized by only one immunoglobulin and T-cell receptor protein. DNA rearrangement is a means for the human body to produce more immunoglobulins and T-cell receptor proteins than there are genes. Changes to the chromatin structure can have an effect on gene expression by modulation of transcription or silencing larger parts of the DNA. In general, for all processes, which happen in the context of higher levels of organization where collections of cells function as a whole, intra-cellular dynamics are linked to inter-cellular coordination of the activity of genomes in different cells. This coordination involves both transient and permanent changes, and must persist over a longer period of time during development.

In the context of intracellular signaling, an example for hybrid modeling is given by the switching phenomena of ERK activities associated with the Raf-1/MEK/ERK pathway (O'Neill and Kolch,
2004; Murphy et al., 2002). Intra-cellular signaling pathways enable cells to perceive changes from their extra-cellular environments and produce appropriate responses (Cho and Wolkenhauer, 2003). Pathways are networks of biochemical reactions but they are also an abstraction biologists use to organize the functioning of the cell; they are the biologist's equivalent of the control engineer's block diagram. The Raf-1/MEK/ERK signaling pathway is a mitogen-activated protein kinase (MAPK) pathway, which exists ubiquitously in most of the eukaryotic cells and is involved in various biological responses (Kolch, 2000). Fig. 3, adapted from O'Neill and Kolch (2004), illustrates the hybrid system dynamics of the Raf-1/MEK/ERK pathway of PC12 cells. The different ERK dynamics are achieved through the combinatorial integration and activation of different Raf isoforms and crosstalk with the cAMP signaling system, which results in discrete state transitions to different cellular dynamics. PC12 cells differentiate in response to nerve growth factor (NGF), but proliferate in response to epidermal growth factor (EGF). Both growth factors utilize the Raf-1/MEK/ERK pathway. The sustained ERK activity caused by the B-raf isoform results in neuronal differentiation while the transient ERK activity caused

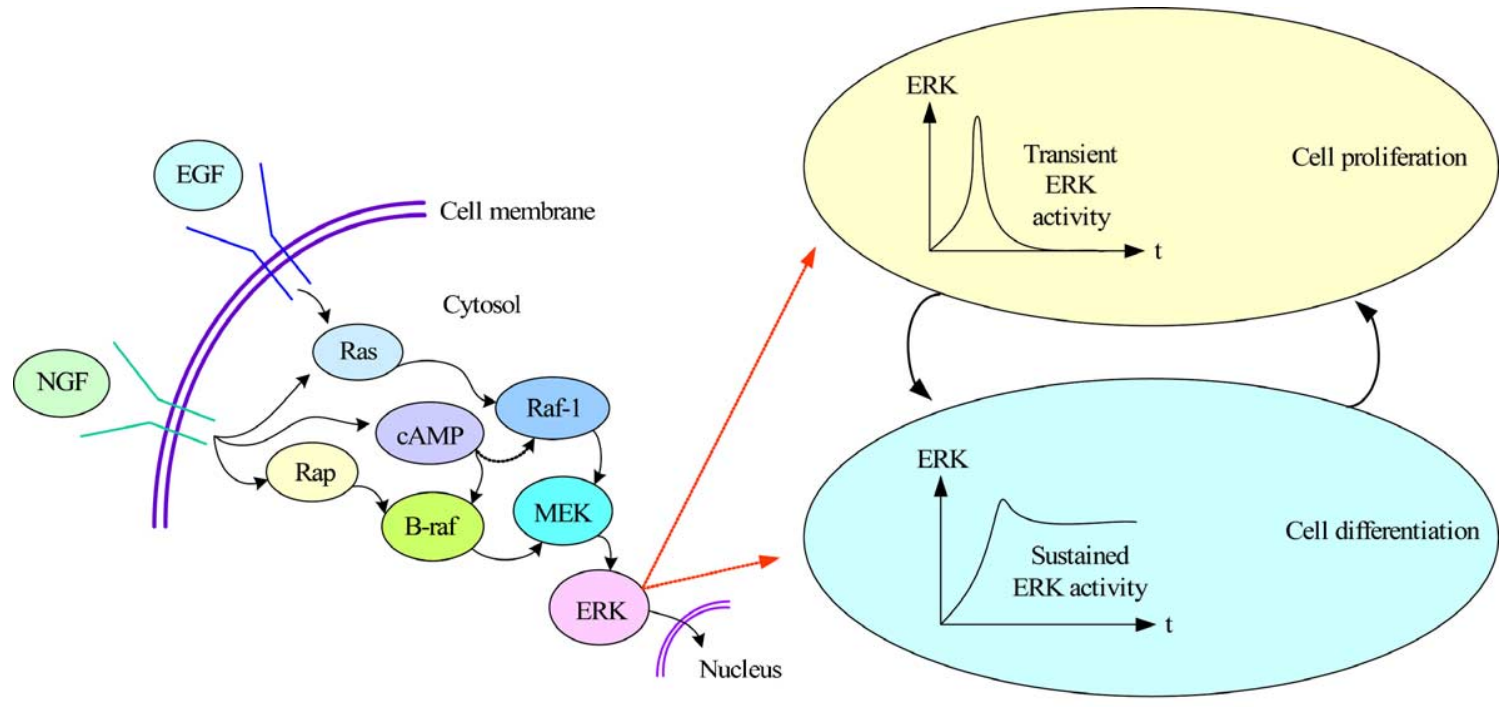

Raf-1/MEK/ERK signaling pathway

Different biological responses

Fig. 3. Hybrid system dynamics of the Raf-1/MEK/ERK cellular signaling pathway in PC12 cells, where both, the quantity and history of ERK concentrations determine discrete state transitions to different dynamics that decide upon the cell's fate. 
by the activation of cAMP signaling and the inhibition of Raf-1 results in cell proliferation.

For more detailed illustration and to provide a comprehensive idea on the hybrid system modeling, we consider a non-autonomous hybrid system model of a xanthophyll cycle reaction system in plant science. Understanding the manner in which excess solar energy is dissipated by photosynthetic membranes under conditions of high light stress has been a major problem facing researchers studying photosynthesis (DemmingAdams, 1990). It has long been known that higher plants possess several defense mechanisms against excess light. Those include the xanthophyll cycle that dissipates excess light energy as heat (Demming-Adams et al., 1996). The xanthophyll cycle is an inter-conversion process between violaxanthin (Vio) and zeaxanthin (Zea). In excessive light, the build up of a transthylakoid proton gradient activates the de-epoxidase, which converts Vio into Zea via an intermediate antheraxanthin (Anth). The back reaction, epoxidation of Zea, is light-independent and catalyzed by an epoxidase thought to be located in the stromal side of the thylakoid membrane (Siefermann and Yamamoto, 1975).

The xanthophyll cycle reaction system is illustrated in Fig. 4, where $x_{1}$ denotes the level of Vio [\%], $x_{2}$ the level of Anth [\%], $x_{3}$ the level of Zea [\%], $k_{1}, k_{2}$ the de-epoxidation rate constants, and $k_{3}, k_{4}$ denote the epoxidation rate constants. The dynamics of the xanthophyll cycle reaction system can be modeled as follows:

$$
\begin{aligned}
& \dot{x}_{1}=-k_{1} x_{1}+k_{4} x_{2} \\
& \dot{x}_{2}=k_{1} x_{1}-k_{4} x_{2}+k_{3} x_{3}-k_{2} x_{2} \\
& \dot{x}_{3}=k_{2} x_{2}-k_{3} x_{3}
\end{aligned}
$$

There have been accumulated studies investigating the dynamics of xanthophyll cycle and the laboratory studies show that the dynamics largely depend on the light stress and the inhibitor treatment such as salicylaldoxime (SA) as an epoxidase inhibitor (Xu et al.,

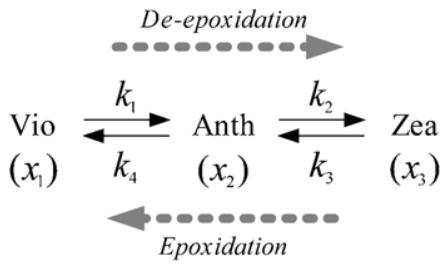

Fig. 4. State diagram of the xanthophyll cycle reaction system.
1999). Hence, the rate constants of the above equations can vary according to the external environmental conditions and the xanthophyll cycle reaction system can be therefore regarded as a hybrid system. We consider a hybrid system model of the xanthophyll cycle reaction system as follows (note that an exposition of the barley leaves is considered but detailed experimental conditions are not described here since those are out of scope of this paper):

$Z_{u}=(Q, X, f, u$, Init, $D, A, C, R)$,

where

- $Q=\left\{q_{1}, q_{2}, q_{3}, q_{4}\right\}$

- $X=\left\{x_{1}, x_{2}, x_{3}\right\}$ and $X=\mathbb{R}^{3}$;

- $f\left(q_{i}, x\right)=\left(-k_{i 1} x_{1}+k_{i 4} x_{2}, k_{i 1} x_{1}-k_{i 4} x_{2}+\right.$ $\left.k_{i 3} x_{3}-k_{i 2} x_{2}, k_{i 2} x_{2}-k_{i 3} x_{3}\right)$, for $i=1,2,3,4$;

- $u \in U=\left\{\left(u_{1}, u_{2}\right)\right\} \quad$ with $u_{1} \in\{\mathrm{LL}, \quad$ HL $\}$ and $u_{2} \in\{+\mathrm{SA},-\mathrm{SA}\}$, where LL denotes the low light stress, HL the high light stress, +SA the inhibitor treatment of SA, and -SA denotes the condition without the treatment of SA;

- Init $=Q \times\left\{x \in \mathbb{R}^{3} \mid 12.2 \leq x_{1} \leq 96.0 \wedge 4.0 \leq\right.$ $\left.x_{2} \leq 25.8 \wedge 0.0 \leq x_{3} \leq 80.9\right\}$;

$D\left(\bar{q}_{1}\right)=\left\{x \in \mathbb{R}^{3} \mid 33.3 \leq x_{1} \leq 96.0 \wedge 4.0 \leq x_{2} \leq\right.$

$\left.6.7 \wedge 0.0 \leq x_{3} \leq 59.9\right\}$,

$D\left(q_{2}\right)=\left\{x \in \mathbb{R}^{3} \mid 33.3 \leq x_{1} \leq 47.7 \wedge 6.7 \leq x_{2}\right.$

$\left.\leq 25.8 \wedge 31.1 \leq x_{3} \leq 59.9\right\}$

$D\left(q_{3}\right)=\left\{x \in \mathbb{R}^{3} \mid 12.2 \leq x_{1} \leq 47.7 \wedge 6.5 \leq x_{2}\right.$ $\left.\leq 21.2 \wedge 31.1 \leq x_{3} \leq 80.6\right\}$,

$D\left(q_{4}\right)=\left\{x \in \mathbb{R}^{3} \mid 12.2 \leq x_{1} \leq 19.7 \wedge 5.1 \leq x_{2}\right.$

$\left.\leq 7.2 \wedge 75.0 \leq x_{3} \leq 80.9\right\}$

- $A=\left\{\left(q_{i}, q_{j}\right) \mid 1 \leq i, \quad j \leq 4, \quad i \neq j\right\}$;

$C: A \rightarrow U$ with $C\left(q_{1}, q_{2}\right)=(\mathrm{LL},-\mathrm{SA})$,

$C\left(q_{1}, q_{3}\right)=(\mathrm{LL},+\mathrm{SA}), \quad C\left(q_{1}, q_{4}\right)=(\mathrm{HL},+\mathrm{SA})$,

$C\left(q_{2}, q_{3}\right)=(\mathrm{LL},+\mathrm{SA}), \quad C\left(q_{2}, q_{1}\right)=(\mathrm{HL},-\mathrm{SA})$,

$C\left(q_{2}, q_{4}\right)=(\mathrm{HL},+\mathrm{SA})$

$C\left(q_{3}, q_{4}\right)=(\mathrm{HL},+\mathrm{SA}), \quad C\left(q_{3}, q_{1}\right)=(\mathrm{HL},-\mathrm{SA})$,

$C\left(q_{3}, q_{2}\right)=(\mathrm{LL},-\mathrm{SA})$,

$C\left(q_{4}, q_{1}\right)=(\mathrm{HL},-\mathrm{SA}), \quad C\left(q_{4}, q_{2}\right)=(\mathrm{LL},-\mathrm{SA})$,

$C\left(q_{4}, q_{3}\right)=(\mathrm{LL},+\mathrm{SA})$

- $R: A \times X \rightarrow P(X)$ with $R\left(q_{i}, q_{j}, x\right)=$

$\{x\}, \quad 1 \leq i, \quad j \leq 4$.

The respective rate constants set of different environmental conditions is as follows: 


\begin{tabular}{lcccc}
\hline & $i=1\left(q_{1}\right)$ & $i=2\left(q_{2}\right)$ & $i=3\left(q_{3}\right)$ & $i=4\left(q_{4}\right)$ \\
\hline$k_{i 1}$ & 11.4 & 14.1 & 17.0 & 19.8 \\
$k_{i 2}$ & 218.7 & 430.6 & 330.1 & 141.9 \\
$k_{i 3}$ & 19.2 & 293.2 & 27.9 & 10.4 \\
$k_{i 4}$ & 7.2 & 33.2 & 10.1 & 9.0 \\
\hline
\end{tabular}

A sample trajectory with the initial state $x(0)=(96.0$, $4.0,0.0)[\%]$ is shown in Fig. 5 according to the execution of the model, where it is assumed that the dark adapted barley leaves are exposed to high light during $[0,0.1]$ (hours), exposed to low light during $[0.1,0.2]$ (hours), SA is added during [0.2, 0.3] (hours), and exposed again to high light during [0.3, 0.4] (hours). It is therefore implied that $C\left(q_{1}, q_{2}\right)=(\mathrm{LL},-\mathrm{SA}), C\left(q_{2}\right.$, $\left.q_{3}\right)=(\mathrm{LL},+\mathrm{SA})$, and $C\left(q_{3}, q_{4}\right)=(\mathrm{HL},+\mathrm{SA})$. From Fig. 5, we realize that in $q_{1}\left(D_{1}\right), x_{3}$, increases rapidly due to the photoprotection mechanism under the high light stress while it decreases for the low light stress in $q_{2}\left(D_{2}\right) ; x_{3}$ increases in $q_{3}\left(D_{3}\right)$ due to inhibition of the epoxidation reaction by SA treatment while it becomes saturated under the further high light stress in $q_{4}\left(D_{4}\right)$.

Other interesting hybrid system modeling examples of biological systems include the full reactive modeling of a multi-cellular animal in Harel (2003) where the

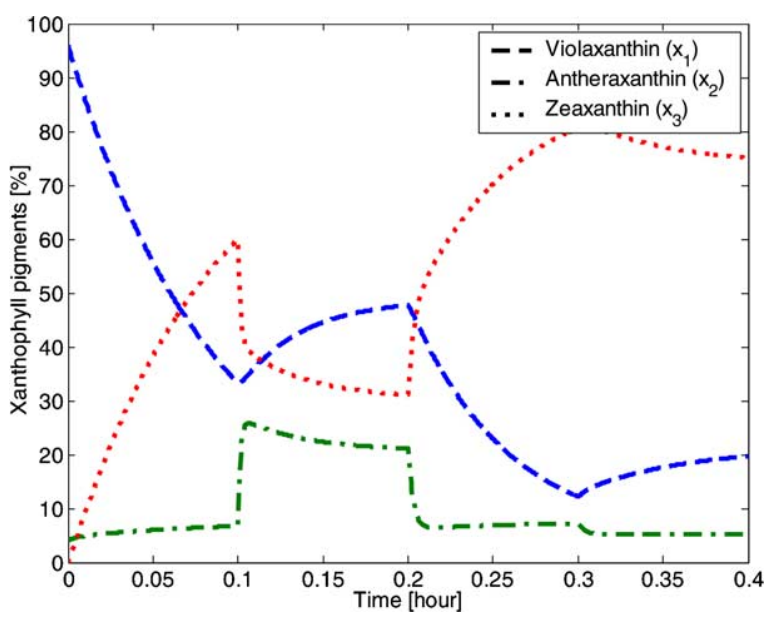

Fig. 5. Sample trajectories of the xanthophyll cycle reaction system according to executions of the hybrid system model.
C. elegans nematode worm is exemplified, the DeltaNotch biological cell signaling networks in Ghosh et al. (2003), and the genetic regulatory network underlying the initiation of sporulation in B. subtilis in De Jong et al. (2003).

\section{Extended hybrid automaton model}

Inspired by Rosen's and Casti's model, and based on the aforementioned dynamical model, we can build an extended hybrid automaton of the hybrid dynamics denoted $M$. It is a minor extension of the model presented in the previous section and defined by:

$M=(Q, X, \Omega, \Gamma, f$, Init, $D, A, C, R)$,

where

- $Q=\left\{q_{\text {normal }}, q_{\text {repaired }}, q_{\text {replicated }}\right\}$

- $X=\mathbb{R}^{n}$ is an open connected set with $X=X_{\mathrm{d}} \cup X_{\mathrm{ud}}$ and $X_{\mathrm{d}} \cap X_{\mathrm{ud}}=\emptyset$;

- $\Omega \subset \mathbb{R}^{m}$;

- $\Gamma \subset \mathbb{R}^{p}$;

- $f=f_{h}\left(x, \omega, \quad \sigma_{l}\right)$ with $x(t) \in X, \omega(t) \in \Omega, \quad$ and $\sigma_{l}=\sigma_{l}(\lambda(t), e)$ in which $\lambda(t) \in \Lambda$ is a time-varying external disturbance and $e \in E_{k}$ is a constant internal control. Here, $\cup_{k \in K} E_{k}$ is a family of controls parameterized in $K \subset \mathbb{N}$ and $\sum=\left\{\sigma_{l}\right\}_{l \in L}$ is a family of environment map parameterized in $L \subset \mathbb{N}$. Moreover, $\gamma=\zeta(x)$ is an output map;

- Init $=\left\{\left(q_{\text {normal }}, x\right) \mid x \in X_{\mathrm{d}}^{0}\right\}$ in which $X_{\mathrm{d}}^{0}$ is the interior of $X_{\mathrm{d}}$

- $D(q)=X_{\mathrm{d}}, \quad$ for $q \in Q$;

- $A=\left\{\left(q_{\text {normal }}, q_{\text {repaired }}\right),\left(q_{\text {repaired }}, q_{\text {repaired }}\right)\right.$, $\left(q_{\text {repaired }}, q_{\text {replicated }}\right),\left(q_{\text {replicated }}, q_{\text {replicated }}\right)$, $\left.\left(q_{\text {replicated }}, q_{\text {normal }}\right)\right\}$;

- $C(a)=X_{\mathrm{ud}}, \quad$ for $a \in A$;

- $R: A \times X \times K \times L \rightarrow P(X) \times K \times L$ is a reset map. Here, we extend the reset map of the hybrid automaton by including two index sets $K, L \subset \mathbb{N}$. The corresponding variables, $k \in K, l \in L$ are simply updated according to $R$ at each discrete transition, similar to the update of the continuous state $x$. So, we have

○ $R\left(q_{\text {normal }}, q_{\text {repaired }}, x, k, l\right)=\left\{\left(x^{\prime}, k, l\right) \mid x^{\prime} \in X\right\}$ (repair),

○ $R\left(q_{\text {repaired }}, q_{\text {repaired }}, x, k, l\right)=\left\{\left(x^{\prime}, k, l\right) \mid x^{\prime} \in X\right\}$ (repair), 


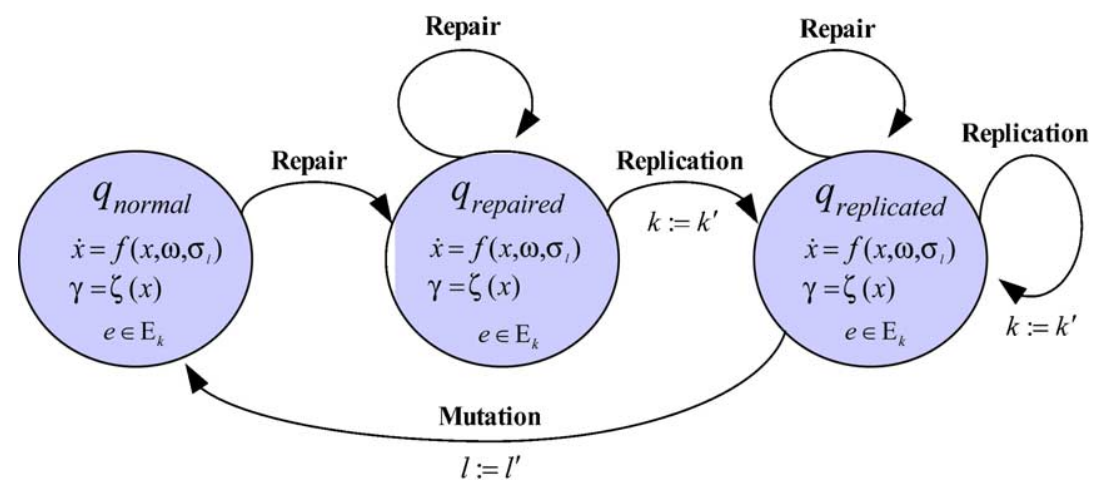

Fig. 6. A hybrid automaton of cellular processes.

○ $R\left(q_{\text {repaired }}, q_{\text {replicated }}, x, k, l\right)=$ $\left\{\left(x^{\prime}, k^{\prime}, l\right) \mid x^{\prime} \in X, k^{\prime} \in K\right\}$ (replication),

○ $R\left(q_{\text {replicated }}, q_{\text {replicated }}, x, k, l\right)=$ $\left\{\left(x^{\prime}, k^{\prime}, l\right) \mid x^{\prime} \in X, k^{\prime} \in K\right\} \quad$ (repair or replication), and

○ $R\left(q_{\text {replicated }}, q_{\text {normal }}, x, k, l\right)=$ $\left\{\left(x^{\prime}, k^{\prime}, l^{\prime}\right) \mid x^{\prime} \in X, k^{\prime} \in K, l^{\prime} \in L\right\}$ (mutation).

Fig. 6 illustrates the hybrid automaton model and Fig. 7 shows three executions accepted by the hybrid automaton of the cellular processes. The execution marked (1) illustrates repair: a discrete transition occurs due to that the continuous state enters $X_{\mathrm{ud}}$. The reset map sets the new continuous state to $x \in X_{\mathrm{d}}$ and a new internal control $e \in E_{k}$. The execution marked (2) illus- trates replication: again a discrete transition occurs due to that the continuous state enters $X_{\mathrm{ud}}$. Now, however, the reset map sets the new continuous state to $x \in X_{\mathrm{ud}}$, which triggers a second transition. This time the reset map sets $x \in X_{\mathrm{d}}$ but also updates $k \in K$. The latter leads to a new $E_{k}$ and $e \in E_{k}$. The execution marked (3) illustrates mutation: here, also the second transition leads to $x \in X_{\text {ud }}$. Therefore, a third transition takes place. Then, the reset map sets $x \in X_{\mathrm{d}}$ and updates $l \in L$, which leads to a new environment variable $\sigma_{l} \in \sum$. Note that the reset map assigns a new continuous state $x^{\prime} \in X$, independent of the past state $x \in X_{\mathrm{ud}}$. Hence, the hybrid automaton accepts several executions and thus represents the uncertainty of the cellular dynamics. Properties such as reachable set computations, liveness, and

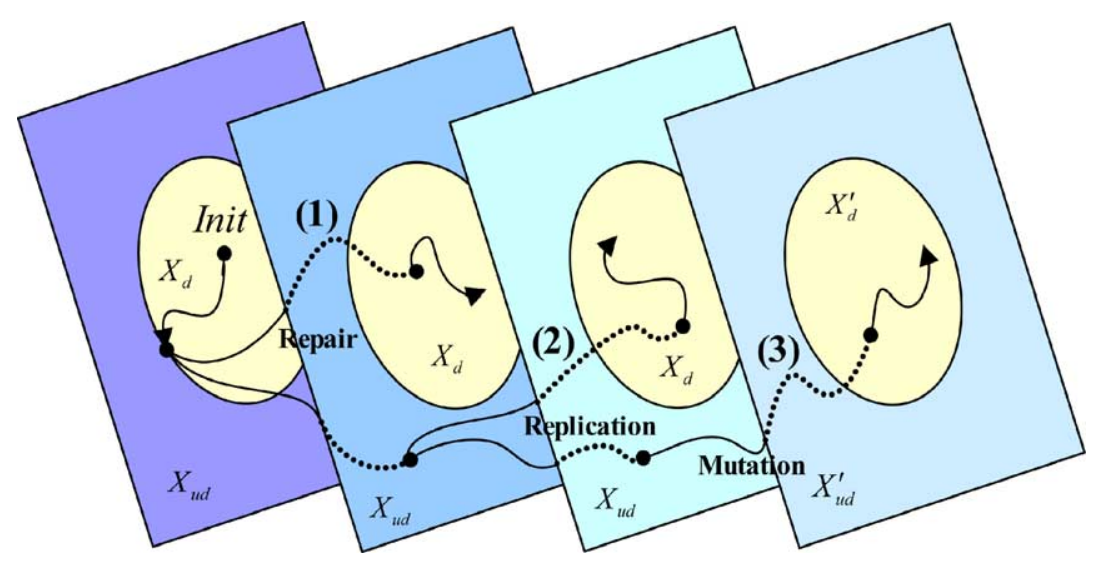

Fig. 7. Examples of executions accepted by the hybrid automaton. 
stability can be analyzed for non-deterministic hybrid automata.

\section{Concluding remarks}

With the availability of technologies that allow us to obtain stimulus-response time series data for modeling and system identification there is going to be an increasing need for conceptual frameworks in which to formulate and test hypotheses about intra- and intercellular dynamics, in general and not just dependent on a particular cell line, cell type, organism, or technology. To this day, experimental data in molecular and cell biology are highly context-dependent but this appears to change and is going to provide control engineers with opportunities and challenges. We here presented an abstract but general, compact mathematical framework that extends $(\mathrm{M}, \mathrm{R})$-theory to take into consideration dynamic aspects of cell signaling and gene expression and to allow for models of reversible, switching and permanent changes occurring. The hybrid automata model illustrated how highly non-linear dynamics and non-determinism can be captured in a formal setting. There exist several tools for the reachability calculations, stability analysis, and computer simulations of hybrid automata, which have been developed over the last decade (Lygeros et al., 2003) and might be useful in the study of cellular processes. The proposed mathematical model of cellular processes could form a basis for further discussions and extensions. One direction of such an extension is the inclusion of the diverse signaling used in cellular communication. Various alternative or complementary concepts could be investigated including temporal logic, concurrency theory, stochastic automata, etc. Any text on modern molecular or cell biology suggests a range of problems for which established system theoretic concepts need to be extended as the complexity of these systems appear to go beyond anything that we have been familiar with in the engineering sciences. A major challenge is the generation of quantitative stimulus-response time series to enable the application of system identification techniques. However, despite of the uncertainty one faces in modeling intracellular dynamics, the encouraging experience is that even drastically simplified models can provide useful practical guidance for the design of experiments, help- ing the experimentalist to decide what to measure and why.

\section{Acknowledgments}

The preparation and revision of the xanthophyll cycle reaction system example benefited from discussion with Prof. C.-H. Lee and S.-Y. Shin. The work of K.H. Cho was supported by a grant from the Korean Ministry of Science and Technology (Korean Systems Biology Research Grant, M10309000006-03B500000211). Karl Henrik Johansson acknowledges the support received by the Swedish Research Council. O. Wolkenhauer acknowledges the support received by the UK Department for the Environment, Food, and Rural Affairs (DEFRA) as part of the M. bovis post-genomics programme.

\section{References}

Alur, R., Dill, D.L., 1994. A theory of timed automata. Theor. Comp. Sci. 126, 183-235.

Alur, R., Henzinger, T.A., Lafferriere, G., Pappas, G.J., 2000. Discrete abstractions of hybrid systems. Proc. IEEE 88, 971984.

Alberts, B., Bray, D., Lewis, J., Raff, M., Roberts, K., Watson, J.D., 2002. Molecular Biology of the Cell, fourth ed. Garland Science.

Asthagiri, A.R., Lauffenburger, D.A., 2001. A computational study of feedback effects on signal dynamics in a mitogen-activated protein kinase (MAPK) pathway model. Biotechnol. Prog. 17, 227-239.

Brown, T.A., 1999. Genomes. ßIOS Scientific Publishers.

Brightman, F.A., Fell, D.A., 2000. Differential feedback regulation of the MAPK cascade underlies the quantitative differences in EGF and NGF signaling in PC12 cells. FEBS Lett. 482, 169-174.

Casti, J.L., 1988. The theory of metabolism-repair systems. Appl. Math. Comput. 28, 113-154.

Cho, K.-H., Wolkenhauer, O., 2003. Analysis and modeling of signal transduction pathways in systems biology. Biochem. Soc. Trans. 31, 1503-1509.

Cho, K.-H., Shin, S.-Y., Kim, H.-Y., Wolkenhauer, O., McFerran, B., Kolch, W., 2003a. Mathematical modeling of the influence of RKIP on the ERK signaling pathway. In: Priami, C. (Ed.), Computational Methods in Systems Biology, LNCS 2602. SpringerVerlag, Berlin, pp. 127-141.

Cho, K.-H., Shin, S.-Y., Lee, H.-Y., Wolkenhauer, O., 2003b. Investigations into the analysis and modeling of the TNF $\alpha$ mediated NF-кB signaling pathway. Genome Res. 13, 2413-2422.

Cho, K.-H., Shin, S.-Y., Kolch, W., Wolkenhauer, O., 2003c. Experimental design in systems biology based on parameter sensitivity analysis with Monte Carlo method: A case study for the TNF $\alpha$ 
mediated NF-кB signal transduction pathway. SIMULATION: Trans. Soc. Model. Simulation Int. 79, 726-739.

De Jong, H., 2002. Modeling and simulation of genetic regulatory systems: a literature review. J. Comput. Biol. 9, 67-103.

De Jong, H., Gouze, J.-L., Hernandez, C., Page, M., Sari, T., Geiselmann, J., 2003. Hybrid modeling and simulation of genetic regulatory networks: a qualitative approach. In: Maler, O., Pnueli, A. (Eds.), Proceedings of the 6th International Workshop on Hybrid Systems: Computation and Control (HSCC 2003), LNCS 2623. Prague, Czech Republic. Springer-Verlag, Berlin, pp. 267-282.

Demming-Adams, B., 1990. Carotenoids and photoprotection in plants: a role for the xanthophyll zeaxanthin. Biochim. Biophys. Acta 1020, 1-24.

Demming-Adams, B., Gilmore, A.M., Adams III, W.W., 1996. In vivo functions of carotenoids in higher plants. FASEB J. 10, $403-412$

Friedman, N., Linial, M., Nachman, I., Pe'er, D., 2000. Using Bayesian networks to analyze expression data. J. Comput. Biol. 7, 585-600.

Ghosh, R., Tiwari, A., Tomlin, C., 2003. Automated symbolic reachability analysis with application to Delta-Notch signaling automata. In: Maler, O., Pnueli, A. (Eds.), Proceedings of the 6th International Workshop on Hybrid Systems: Computation and Control (HSCC 2003), LNCS 2623. Prague, Czech Republic. Springer-Verlag, Berlin, pp. 233-248.

Harel, D., 2003. A grand challenge for computing: full reactive modeling of a multi-cellular animal. Bull. EATCS Eur. Assoc. Theor. Comp. Sci. 81, 226-235.

Heinrich, R., Neel, B.G., Rapoport, T.A., 2002. Mathematical models of protein kinase signal transduction. Mol. Cell 9, 957-970.

Iglesias, P.A., Levchenko, A., 2002. Modeling the cell's guidance system. Science STKE 148, re12 (www.stke.org/cgi/content/ full/sigtrans;2002/148/re12).

Jordan, M.I. (Ed.), 1996. Learning in Graphical Models. Kluwer Academic Publishers, Dordrecht, The Netherlands.

Kholodenko, B.N., Klyatkin, A., Bruggeman, F.J., Sontag, E., Westerhoff, H.V., Hoek, J.B., 2002. Untangling the wires: a strategy to trace functional interactions in signaling and gene networks. PNAS 99, 12841-12846.

Kolch, W., 2000. Meaningful relationships: the regulation of the Ras/Raf/MEK/ERK pathway by protein interactions. Biochem. J. 351, 289-305.

Lygeros, J., Johansson, K.H., Simic, S.N., Zhang, J., Sastry, S., 2003. Dynamical properties of hybrid automata. IEEE Trans. Automatic Control 48, 2-17.

Maki, Y., Tominaga, D., Okamoto, M., Watanabe, S., Eguch, Y., 2001. Development of a system for the inference of large scale genetic networks. Proc. Pacific Symp. Biocomput. 6, 446458.
Murphy, L.O., Smith, S., Chen, R.H., Fingar, D.C., Blenis, J., 2002. Molecular interpretation of ERK signal duration by immediate early gene products. Nat. Cell Biol. 4, 556-564.

O'Neill, E., Kolch, W., 2004. Conferring specificity of the ubiquitous Raf/MEK signaling pathway. Br. J. Cancer 90, 283-288.

Paulsson, J., 2004. Summing up the noise. Nature 427, 415-418.

Rao, C.V., Wolf, D.M., Arkin, A.P., 2002. Control, exploitation and tolerance of intracellular noise. Nature 420, 231-237.

Rosen, R., 1971. Some realizations of (M,R)-systems and their interpretation. Bull. Math. Biophys. 33, 303-319.

Rzhetsky, A., Iossifov, I., Koike, T., Krauthammer, M., Kra, P., Morris, M., Yu, H., Duboue, P.A., Weng, W., Wilbur, W.J., Hatzivassiloglou, V., Friedman, C., 2004. GeneWays: a system for extracting, analyzing, visualizing, and integrating molecular pathway data. J. Biomed. Inform. 37, 43-53.

Schoeberl, B., Eichler-Jonsson, C., Gilles, E.D., Müller, G., 2002. Computational modeling of the dynamics of the MAP kinase cascade activated by surface and internalized EGF receptors. Nat. Biotechnol. 20, 370-375.

Shmulevich, I., Dougherty, E.R., Kim, S., Zhang, W., 2002. Probabilistic Boolean network: a rule-based uncertainty model for gene regulatory networks. Bioinformatics 18, 261-274.

Siefermann, D., Yamamoto, H.Y., 1975. Properties of NADPH and oxygen-dependent zeaxanthin epoxidation in isolated chloroplasts. Arch. Biochem. Biophys. 171, 70-77.

Smolen, P., Baxter, D.A., Byrne, J.H., 2000. Modeling transcriptional control in gene networks-methods, recent results, and future directions. Bull. Math. Biol. 62, 247-292.

Swameye, I., Müller, T.G., Timmer, J., Sandra, O., Klingmüller, U., 2003. Identification of nucleocytoplasmic cycling as a remote sensor in cellular signaling by databased modeling. PNAS 100, 1028-1033.

Tyson, J.J., Chen, K.C., Novak, B., 2003. Sniffers, buzzers, toggles and blinkers: dynamics of regulatory and signaling pathways in the cell. Curr. Opin. Cell Biol. 15, 221-231.

Tegner, J., Young, M.K.S., Hasty, J., Collins, J.J., 2003. Reverse engineering gene networks: integrating genetic perturbations with dynamical modeling. PNAS 100, 5944-5949.

Wolf, D.M., Arkin, A.P., 2003. Motifs, modules and games in bacteria. Curr. Opin. Microbiol. 6, 125-134.

Wolkenhauer, O., 2001. Systems biology: the reincarnation of systems theory applied in biology? Brief. Bioinform. 2, 258-270.

Wolkenhauer, O., Kitano, H., Cho, K.-H., 2003. Systems biology: looking at opportunities and challenges in applying systems theory to molecular and cell biology. IEEE Control Syst. Mag. 23, 38-48.

Xu, C.C., Jeon, Y.A., Hwang, H.J., Lee, C.-H., 1999. Suppression of zeaxanthin epoxidation by chloroplast phosphatase inhibitors in rice leaves. Plant Sci. 146, 27-34. 